\title{
Supporting Information for Coatings releasing the relaxin peptide analogue B7-33 reduce fibrotic encapsulation
}

Nicholas G. Welch ${ }^{1}$, Shayanti Mukherjee ${ }^{2}$, Mohammed A. Hossain ${ }^{3}$, Praveen Praveen ${ }^{3}$, Jerome A. Werkmeister ${ }^{2}$, John D. Wade ${ }^{3,4}$, Ross A. D. Bathgate ${ }^{3,5}$, David A. Winkler ${ }^{1,6,7,8}$, Helmut Thissen $^{1 *}$

${ }^{1}$ CSIRO Manufacturing, Research Way, Clayton, VIC 3168, Australia.

${ }^{2}$ The Ritchie Centre, Hudson Institute of Medical Research, Clayton, VIC 3168, Australia.

${ }^{3}$ The Florey Institute of Neuroscience and Mental Health, Parkville, VIC 3052, Australia.

${ }^{4}$ School of Chemistry, The University of Melbourne, VIC 3010, Australia.

${ }^{5}$ Department of Biochemistry and Molecular Biology, The University of Melbourne, VIC 3010, Australia

${ }^{6}$ La Trobe Institute for Molecular Science, La Trobe University, Kingsbury Drive, Bundoora, VIC 3083, Australia.

${ }^{7}$ Monash Institute of Pharmaceutical Sciences, Royal Parade, Parkville, VIC 3052, Australia.

${ }^{8}$ School of Pharmacy, The University of Nottingham, Nottingham NG7 2RD, UK.

* Corresponding author's email address: Helmut.Thissen@csiro.au 


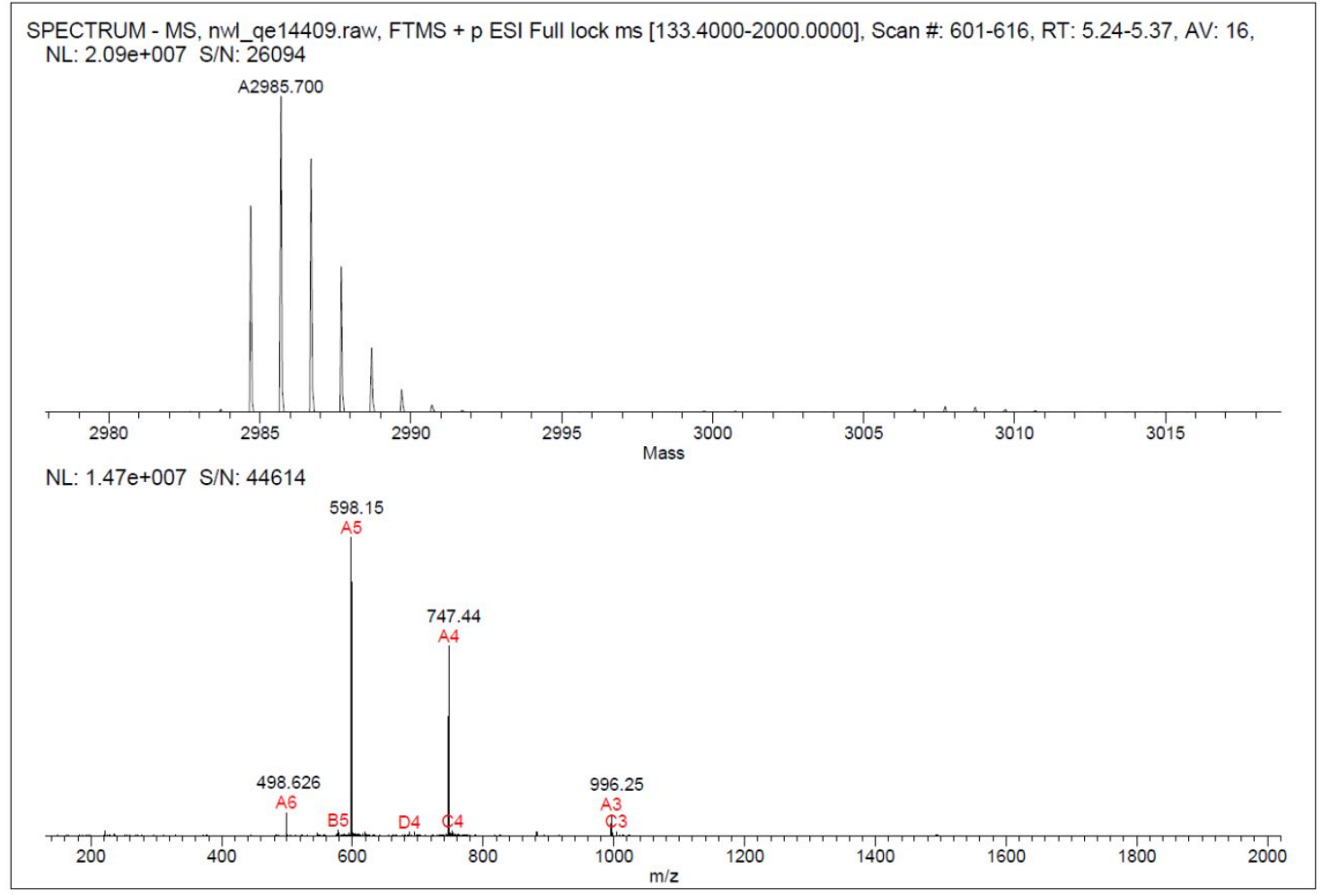

Figure S1 | Deconvoluted spectra of HPLC-MS electrospray ionisation measurement of the B7-33 peptide in water showing the molecular ion 2985.7 Da (top panel) and the associated molecular ions fragments at 996.25, 747.44 and $598.15 \mathrm{~m} / \mathrm{z}$ corresponding to the $[\mathrm{M}+3 \mathrm{H}]^{3+}$, $[\mathrm{M}+4 \mathrm{H}]^{4+}$, and $[\mathrm{M}+5 \mathrm{H}]^{5+}$ (bottom panel). 

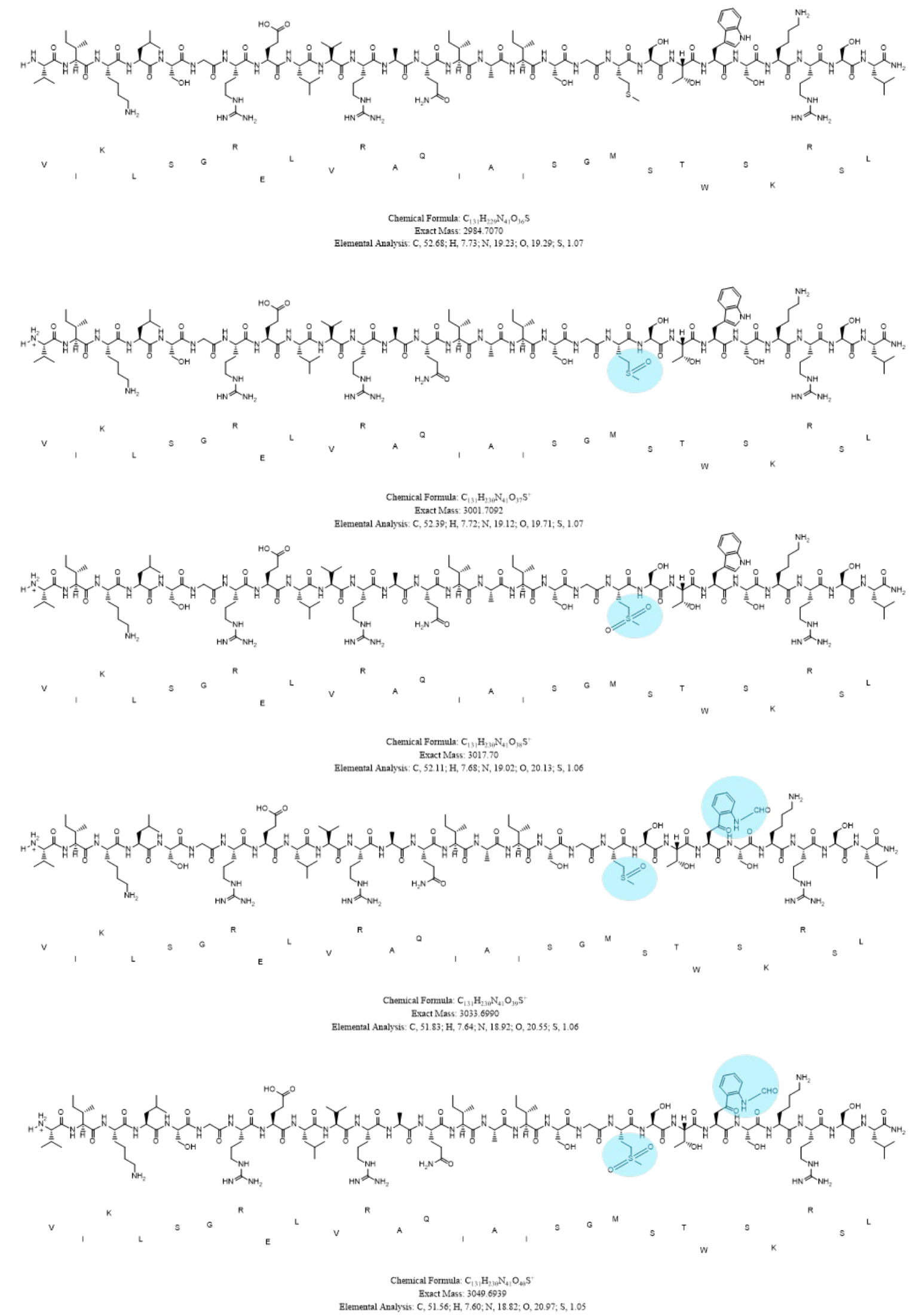

Figure $\boldsymbol{S 2} \mid$ B7-33 and the proposed oxidized forms observed using the MALDI technique. 